05

\title{
Пороговые энергии атомных смещений в $\alpha$-Fе под деформацией: моделирование методом молекулярной динамики
}

\author{
(C) М.Ю. Тихончев ${ }^{1}$, В.В. Светухин ${ }^{1,2}$ \\ ${ }^{1}$ Научно-исследовательский технологический институт им. С.П. Капицы \\ Ульяновского государственного университета, Ульяновск \\ ${ }^{2}$ Институт нанотехнологий микроэлектроники РАН, Москва \\ E-mail: tikhonchev@sv.ulsu.ru
}

Поступило в Редакцию 30 октября 2016 г.

Путем молекулярно-динамического моделирования проведено исследование влияния деформаций на среднюю пороговую энергию смещения в $\alpha$-Fe. Рассмотрены гидростатическая и несколько одноосных сжимающих и растягивающих деформаций. Показано, что значение пороговой энергии смещения хорошо описывается линейной зависимостью от вызванного деформацией относительного изменения объема. Угловой коэффициент линейной аппроксимации составил $-1.21 \mathrm{eV}$ для изменений объема в процентах. Изменения в энергии смещения при деформациях, сохраняющих объем, незначительны.

DOI: 10.21883/PJTF.2017.07.44469.16548

Одной из важных характеристик радиационного воздействия, используемых при исследовании изменений физических и механических свойств материалов при каскадообразующем облучении, является так называемая повреждающая доза. Эта величина выражается в количестве атомных смещений в кристаллической решетке материала, приходящихся на один атом (сна - смещение на атом). Знание повреждающей дозы позволяет сравнивать различные условия облучения и прогнозировать поведение материала в действующих и в проектируемых реакторах. Общепризнанной процедурой подсчета числа атомных смещений является NRT-стандарт [1]. Важной величиной, используемой для подсчета сна, является эффективная пороговая энергия смещения (или просто - энергия смещения). Именно эта величина несет в себе практически всю информацию о кристаллической решетке при использовании NRT-стандарта. Оценки пороговых энергий смещения получают 
в экспериментах путем облучения электронами тонких монокристаллических фольг [2] или рассчитывают путем атомистического моделирования методом молекулярной динамики (МД-моделирование) $[3,4]$. Достаточно подробный обзор методических особенностей применения МД-моделирования для оценки энергий смещения дан Нордлундом (Nordlund) и др. [3]. Хорошо известно, что значение энергии смещения существенно зависит от кристаллографического направления импульса атома отдачи. Большое значение имеют минимальная $\left(E_{d}^{\min }\right)$ и средняя $\left(E_{d}\right)$ пороговые энергии смещения: $E_{d}^{\min }$ надежно определяется в экспериментах и может использоваться для тестирования потенциалов межчастичного взаимодействия, $E_{d}$ используется в NRT-стандарте для подсчета числа атомных смещений. Согласно работе [3], величина $E_{d}$ может определяться либо как среднее значение энергии смещения на множестве всех направлений $\left(E_{d}^{a v}-\right.$ средняя пороговая энергия), либо как значение энергии, обеспечивающее вероятность образования стабильной пары Френкеля (Frenkel pair) $p=1 / 2$ при случайном равномерном выборе пространственного направления $\left(E_{d}^{\text {med }}-\right.$ медианная пороговая энергия). При этом в [3] были получены близкие значения $E_{d}^{a v}$ и $E_{d}^{\text {med }}$ для $\alpha$-Fe для 12 различных потенциалов межчастичного взаимодействия.

Кроме облучения реакторные материалы в процессе эксплуатации подвержены также деформациям под механическими нагрузками. Большинство экспериментальных исследований влияния нагружения на особенности радиационного повреждения материала проведено для сталей аустенитного класса [5-7]. Имеется лишь небольшое количество работ [8-11], посвященных изучению влияния нагружения и деформации $\alpha$-Fe на параметры его первичного радиационного повреждения. Во всех этих работах исследования проведены путем МД-моделирования. Билер и др. [11] недавно исследовали влияние деформации и температуры на пороговую энергию смещения $\left(E_{d}^{\min }\right.$ и $\left.E_{d}^{m e d}\right)$ в $\alpha$-железе. Были рассмотрены гидростатическое расширение $2 \%$ и тетрагональный сдвиг $5 \%$ при температурах 300 и $500 \mathrm{~K}$. Было установлено снижение энергии смещения при рассмотренных деформациях. Причем наибольшее снижение наблюдалось при гидростатическом расширении и составило около $25 \%$ при обеих температурах. Сохраняющий объем тетрагональный сдвиг приводит к существенно меньшим (в 2-4 раза) изменениям $E_{d}^{m e d}$. Следует отметить, что рассмотренные в [11] деформации являются слишком большими с точки зрения практических приложений.

Письма в ЖТФ, 2017, том 43, вып. 7 
$\mathrm{B}$ настоящей работе изучается влияние деформации кристалла $\alpha$-Fe на величину средней пороговой энергии смещения. Метод исследования - МД-моделирование. Рассмотрены всесторонняя (гидростатическая) и несколько одноосных деформаций величиной $0.1,0.5$ и $1 \%$ обоего знака. При этом кристаллит всегда остается в области упругих деформаций.

Расчеты проводились методом молекулярной динамики с многочастичным полуэмпирическим потенциалом Акланда, Менделева (Ackland, Mendelev) и др. [12]. Этот потенциал был разработан в 2004 г. и остается к настоящему времени одним из наиболее удачных и широко используемых для атомистического моделирования $\alpha$-железа. В частности, в работах $[3,13]$ было показано, что он хорошо воспроизводит известные экспериментальные оценки пороговой энергии смещения для низкоиндексных кристаллографических направлений и обеспечивает значение $E_{d}^{a}$, близкое к $40 \mathrm{eV}$, рекомендуемое стандартом ASTM [14]. Моделирование проводили, используя ОЦК-кристаллиты, имеющие форму прямоугольного параллелепипеда, близкого к кубу, с периодическими граничными условиями. Число атомов в системе - от 10404 до 12096. Поскольку работа нацелена на исследование только эффекта деформации, все расчеты проводятся при нулевой начальной температуре. Деформацию задавали путем соответствующего изменения линейных размеров кристаллита. Одноосную деформацию задавали для четырех кристаллографических направлений [001], [111], [112] и [210]. Кроме собственно одноосной деформации, когда изменяется линейный размер кристаллита вдоль только одного направления, рассматривали одноосную деформацию с сохранением объема, когда изменение объема компенсировалось соответствующим изменением линейного размера противоположного знака вдоль осей, перпендикулярных основной оси нагружения. Вычисления проводили по численной схеме Верле (Verlet) [15] с неравномерным временным шагом численного интегрирования, который выбирался так, чтобы атомы системы смещались за один шаг не более чем на $0.02 \AA$ и величина шага не превосходила $2 \mathrm{fs}$. Критерий устойчивого смещения - наличие точечных дефектов в кристаллите после 8 ps с момента вылета атома отдачи. Точечные дефекты определяли методом ячеек Вигнера-Зейтца (Wigner-Seitz): пустая ячейка трактуется как вакансия, ячейка, содержащая более одного атома, - как междоузельная конфигурация. При нахождении $E_{d}$, начиная с небольших энергий $(12 \mathrm{eV})$, повышали энергию атома отдачи 


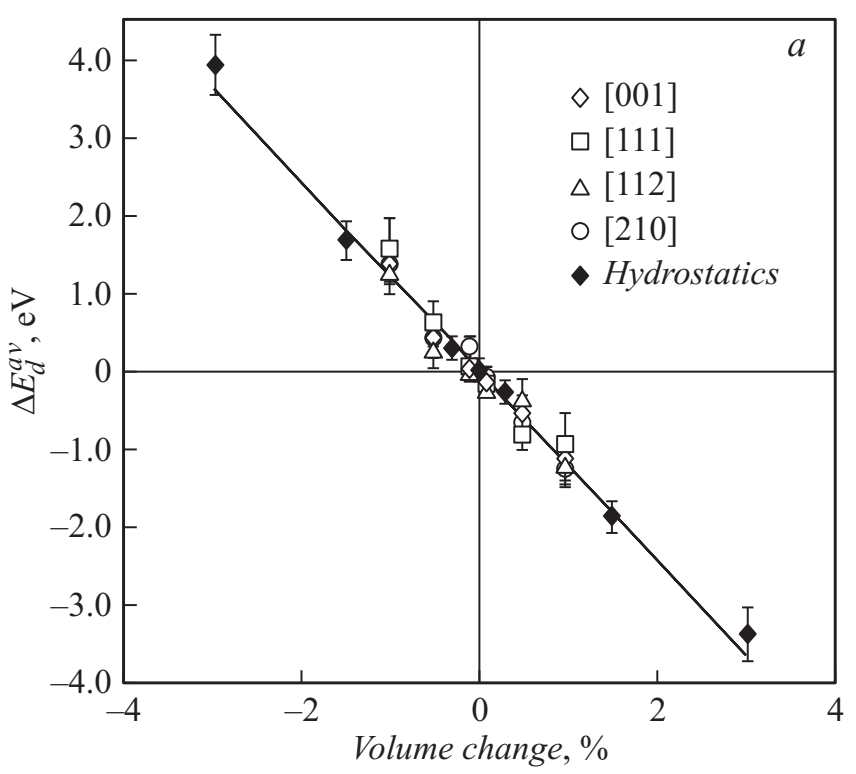

Изменение средней пороговой энергии смещения при деформациях, изменяющих $(a)$ и сохраняющих $(b)$ объем кристалла.

с шагом $1 \mathrm{eV}$, пока не достигали значения, при котором в кристаллите образуются устойчивые пары Френкеля (Frenkel). Направление импульса атома отдачи выбирали путем моделирования случайного изотропного вектора. Для каждого направления оценивали $E_{d}$ в недеформированном и деформированных состояниях и находили абсолютное изменение пороговой энергии при деформации. Полученные результаты усредняли по всем рассмотренным направлениям, получая, таким образом, оценку изменения средней энергии смещения $\Delta E_{d}^{a v}$. Для каждого нагружения моделировали по 400 направлениям атома отдачи. Общее число промоделированных вариантов с учетом перебора энергий атома отдачи составило более полумиллиона.

Оценка средней пороговой энергии смещения в отсутствие деформации составила $E_{d}^{a v}=39.3 \pm 0.5 \mathrm{eV}$ (здесь и далее приводятся статистические погрешности, равные одному стандартному отклонению для выборочного среднего $(1 \sigma))$. Результаты для рассмотренных де-

Письма в ЖТФ, 2017, том 43, вып. 7 


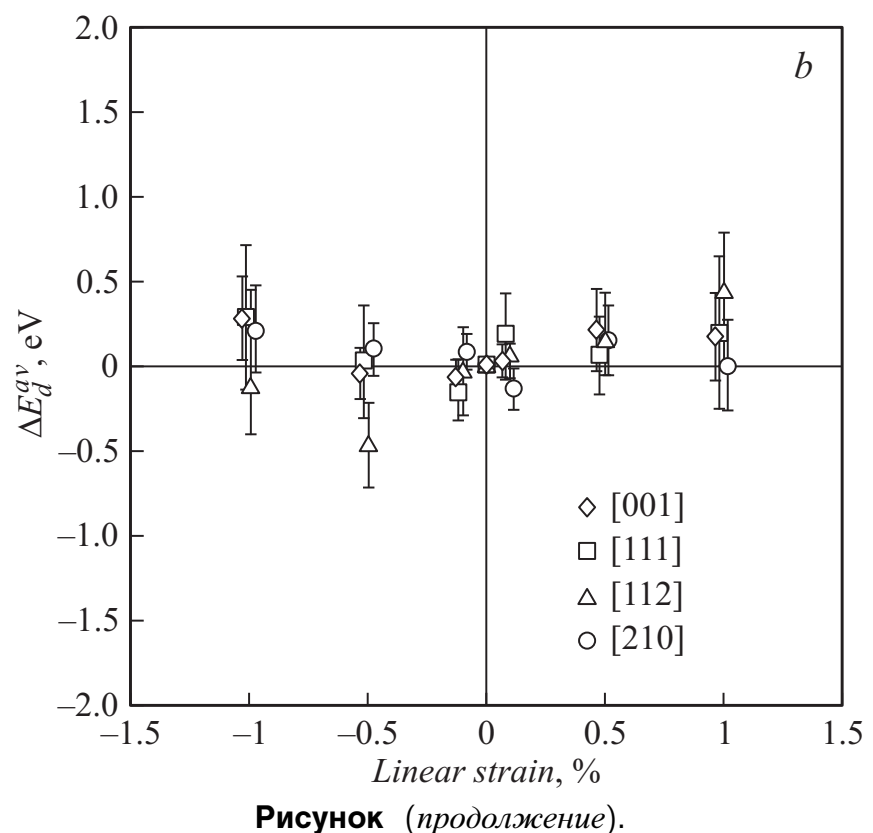

формаций, не сохраняющих объем, представлены на рисунке, $a$. Хорошо видно, что энергия смещения растет при сжимающей деформации и снижается при растягивающей. Независимо от типа и знака деформации она хорошо описывается линейной зависимостью от изменения объема

$$
E_{d}^{a v}(\Delta V)=k \Delta V+39.3 \mathrm{eV},
$$

где $\Delta V$ - относительное изменение объема при деформации. Значение углового коэффициента, полученное методом наименьших квадратов, составило $k=-1.21 \mathrm{eV}$ (для $\Delta V$ в \%). Экстраполируя полученную зависимость на случай гидростатической деформации $2 \%$, что соответствует $\Delta V \approx 6 \%$, получим относительное изменение энергии смешения $\sim 19 \%$. Это немногим меньше изменения в $25 \%$, полученного ранее в работе [11].

На рисунке, $b$ представлены результаты расчетов для сохраняющих объем одноосных деформаций. Полученные оценки $\Delta E_{d}^{a v}$ лежат здесь 
в пределах $\pm 0.5 \mathrm{eV}$ или $\pm 1.3 \%$, т. е. являются небольшими. Почти все вызванные деформацией изменения энергии смещения не выходят при этом за пределы погрешности расчета. Таким образом, влияние сохраняющих объем деформаций на значение $E_{d}^{a v}$ является незначительным.

Полученные результаты позволяют сделать следующие выводы для рассмотренных величин деформации. На изменение пороговой энергии смещения, прежде всего, влияет изменение объема деформированного кристалла. Значение $E_{d}^{a v}$ хорошо описывается одной линейной зависимостью от относительного изменения объема для всех рассмотренных сжимающих и растягивающих деформаций. Деформации, сохраняющие объем, не влекут хорошо видимых изменений энергии смещения. Следует отметить, что изменение $E_{d}^{a v}$ при деформациях менее $0.5 \%$ не превышает $2 \mathrm{eV}$. Это небольшое изменение. Поэтому влияние практически важных величин деформации на величину пороговой энергии смещения можно считать незначительным.

Работа выполнена при поддержке Министерства образования и науки РФ в рамках государственного задания вузам, а также при частичной поддержке РФФИ (проект 16-01-00542).

\section{Список литературы}

[1] Norgett N.J., Robinson M.T., Torrens I.M. // Nucl. Eng. Design. 1975. V. 33. P. 50-56.

[2] Vajda P. // Rev. Modern Phys. 1977. V. 49. N 3. P. 481-521.

[3] Nordlund K., Wallenius J., Malerba L. // Nucl. Instr. Meth. B. 2006. V. 246 (2). P. 322-332.

[4] Кирсанов В.В. // ФММ. 1996. Т. 81. В. 2. С. 36-39.

[5] Bates J.F., Gilbert E.R. // Ibid. 1978. V. 71. P. 286-292.

[6] Неустроев В.С., Белозеров С.В., Макаров Е.И., Обухов А.В. // ФММ. 2014. T. 115 . № 10. C. 1070.

[7] Марголин Б.3., Мурашова А.И., Неустроев В.С. // Вопросы материаловедения. 2011. № 4 (68). С. 124-139.

[8] Корчуганов А.В., Зольников К.П., Крыжевич Д.С. и др. // Вопросы атомной науки и техники: Термоядерный синтез. 2015. Т. 38 (1). С. $42-48$.

[9] Gao F., Bacon D., Flewitt P., Lewis T. // Nucl. Instr. Meth. B. 2001. V. 180. P. 187-193.

[10] Beeler B., Asta M., Hosemann P., Grønbech-Jensen N. // J. Nucl. Mater. 2015. V. 459. P. 159-165.

Письма в ЖТФ, 2017, том 43, вып. 7 
[11] Beeler B., Asta M., Hosemann P. et al. // Nucl. Mater. 2016. V. 474. P. 113-119.

[12] Ackland G.J., Mendelev M.I., Srolovitz D.J. et al. // J. Phys.: Condens. Matter. 2004. V. 16. P. S2629-S2642.

[13] Tikhonchev M., Svetukhin V., Kadochkin A., Gaganidze E. // J. Nucl. Mater. 2009. V. 395. P. 50-57.

[14] ASTM E521, (E521-89). Annual Book of ASTM Standards. 1995. V. 12.02.

[15] Verlet L. // Phys. Rev. 1967. V. 159. P. 98-103. 